\title{
Los Modelos cambiantes de la Transferencia y sus Implicaciones Clínicas: Supuestos Residuales que Difícilmente Mueren ${ }^{\mathrm{a}}{ }^{1}$
}

\author{
James L. Fosshage, Ph.D ${ }^{b}$ \\ I.A.P.S.P. , New York.
}

\begin{abstract}
La transferencia es sin duda el concepto más importante del psicoanálisis, hace referencia a los sentimientos y fantasías directas del paciente en relación al analista, alberga una teoría etiológica como fuente de estos sentimientos y fantasías, contiene una teoría de acción terapéutica, e incluye una teoría respecto a la práctica clínica. La transferencia ha evolucionado hacia diferentes conceptualizaciones, concepciones y definiciones desde la formulación inicial de Freud (1895) hace ya más de un siglo. Mientras que los modelos de la transferencia pueden variar en algunos de los aspectos que acabo de introducir, dando lugar de este modo a un amplio abanico de paradigmas, consideramos que a un nivel de estructura básico todas las teorías acerca de la transferencia son representadas por uno de los tres modelos fundamentales- el modelo del desplazamiento, el modelo inclusivo y la transferencia como modelo organizador. Esta conferencia muestra esos modelos².
\end{abstract}

Palabras clave: Transferencia, Modelo Freudiano, Modelo Inclusivo, Modelo Relacional

Transference is arguably the most pivotal concept in psychoanalysis, for it addresses the patient's immediate feelings and fantasies toward the analyst, it houses an etiological theory as to the source of these feelings and fantasies, it contains a theory of therapeutic action, and it encompasses a theory of clinical practice. Transference has evolved in various conceptualizations, understandings and definitions since Freud's (1895) initial formulation over a century ago. While models of transference can vary in any one of the above areas, creating a considerable array of models, we believe that all transference theories at a basic structural level are represented by one of three fundamental models-the displacement, totalist and organizing models of transference. This lecture displays those models.

Key Words: Transference, Freudian model, Inclusive Model, Relational Model. English Title: Changing Models of Transference and their clinical implications: Residual Assumptions Die Hard.

\section{Cita bibliográfica / Reference citation:}

Fosshage, J.L. (2016). Los modelos cambiantes de la Transferencia y sus implicaciones clínicas: Supuestos residuales que difícilmente mueren. Clínica e Investigación Relacional, 10 (2): 373-392. [ISSN 1988-2939] [Recuperado de www.ceir.info ] DOI: 10.21110/19882939.2016.100203

\footnotetext{
a Texto de la conferencia leída por el Prof. James I. Fosshage en el Instituto de Psicoterapia Relacional / Ágora Relacional, Madrid, 6 de Mayo de 2016. Traducción de Andrea Iturriaga Medrano, con comentarios añadidos traducidos por N. Monserrat Gómez, que se incluyen como notas al final.

b James L. Fosshage, PhD, es ExPresidente de la International Association of Psychoanalytic Self Psychology y el anterior presidente Association for Autonomous Psychoanalytic Institutes (AAPI). Es el co-fundador y director del consejo del National Institute for the Psychotherapies (NYC), miembro facultativo fundador del Institute for the Psychoanalytic Study of Subjectivity (NYC), y profesor de Psicología Clínica en el Programa Postdoctoral de Psicoterapia y Psicoanálisis de la Universidad de Nueva York. Entre sus libros están: Dream interpretation: $A$ comparative study, edición revisada, en el que es co-autor junto con Clemens Loew, Self and Motivational Systems: Toward a theory of Psychoanalitic Technique, The Clinical Exchange: Technique from the Standpoint of Self and Motivational Systems, and Spirit of Inquiry: Communication in Psychoanalysis, todos ellos con la colaboración como co-autores de Joseph Lichtenberg y Frank Lachmann. Actualmente tiene su propia consulta privada como psicoanalista y psicoterapeuta en New York City y en Tenafly, New Jersey.
}

CelR Vol. 10 (2) - Junio 2016 ISSN 1988-2939 - www.ceir.info

@ Derechos reservados/Copyright de Clínica e investigación Relacional y los autores. Prohibida la reproducción total o parcial sin autorización expresa. Este material es para uso científico y profesional exclusivamente y puede contener información clínica sensible. Los editores no se responsabilizan de los contenidos de los autores. Dirigir las consultas sobre derechos y autorizaciones a ceir@psicoterapiarelacional.es 
En la evolución y conceptualización de los tres modelos básicos sobre la Transferencia [el modelo del desplazamiento (Freudiano), el modelo inclusivo (Gill) y la transferencia como modelo organizador], ha sido fundamental la todavía en curso, aunque fluctuante, integración de dos cambios paradigmáticos que comenzaron hace 60 años dentro de las ciencias y el psicoanálisis - estos son el cambio desde objetivismo al constructivismo y desde la teoría intrapsíquica a la teoría relacional. En primer lugar, el cambio epistemológico dentro del psicoanálisis ha conllevado un profundo impacto tanto teórico como clínico. Inicialmente derivadas de la epistemología objetivista; las transferencias eran entendidas como distorsiones de la realidad; posteriormente surgiendo de la epistemología relativista (Lo que Orange, 1995, ha denominado perspectivismo realista), las transferencias son vistas actualmente como construcciones de "la realidad". En segundo lugar, cuando las transferencias, desde el punto de vista del desarrollo y actualmente entendidas dentro de la relación terapéutica, son vistas principalmente como resultado de fuentes intrapsíquicas o desde el propio entramado relacional, estas explicaciones etiológicas, tan esencialmente diferentes respecto de la transferencia, tienen un enorme impacto en la comprensión, las explicaciones interpretativas y las conceptualizaciones de la acción terapéutica. Al integrar estos dos cambios de paradigmas podemos ver ahora el encuentro analítico como co-creado y co-constuido en la interacción de dos subjetividades que dan lugar al campo intersubjetivo (Atwood y Storolow, 1084; Storolow, Brandchaft y Atwood, 1987) o relacional (Greenberg y Mitchell, 1983; Mitchell, 1988). 3 Al hablar dentro del ámbito de la transferencia y contratransferencia, este término acerca de la implicación de la contratransferencia del analista en reacción a la transferencia no recoge adecuadamente la complejidad de la interacción analista-paciente. Recientemente, en la aplicación de la teoría de los sistemas hacemos referencia al sistema diádico paciente-analista. En este trabajo nos centraremos en la conceptualización del fenómeno de la transferencia, entendiendo que dentro de una teoría de campo (y sistemas) este fenómeno puede atribuirse al paciente y al analista por igual (esto será desarrollado). ${ }^{4}$

Sumado a estas transformaciones de paradigmas, los descubrimientos y teorías empíricas provenientes de la ciencia cognitiva, la teoría de los sistemas, las investigaciones dentro de la cognición social y la neurociencia cognitivo/afectiva han contribuido a su vez a una comprensión más detallada y delimitada del fenómeno de la transferencia (Wachtel, 1980; Hoffman, 1983, 1991; Storolow/Lachmann, 1984/85; Fosshage, 1994; Lichtenberg, Lachmann y Fosshage, 1996; Weston y Gabbard, 2002; Schachter, 2002; entre otros). Expresándolo en un sentido más amplio, todos estos discursos científicos clarifican y expanden nuestro entendimiento acerca de la formación y transformación de procesos organizadores (Fosshage, 2013). Aunque las implicaciones clínicas de estos nuevos conocimientos son revolucionarias cuanto menos, su descripción, integración y la 
consistencia de su uso clínico están todavía, según nuestro punto de vista, en proceso. Mientras que la integración de cambios teóricos conlleva un proceso gradual, la integración de implicaciones clínicas que surgen de innovaciones teóricas requiere aún más tiempo para que sus actitudes y supuestos clínicos sean duraderos $y$, tal y como ocurre en todas las organizaciones psicológicas ya consolidadas, pasan a operar automáticamente como procedimientos implícitos y explícitos que mantienen la organización psicológica, seguridad en el sentido del "conocer", y un fiable sentido de la competencia, siendo estos aspectos resistentes al cambio. 5

Mi intención en la ponencia de esta noche es centrarme más específicamente en el supuesto técnico del psicoanálisis tradicional que supone que el foco principal, sino exclusivo, se centra en la transferencia de la relación analítica. Tanto si la transferencia puede observarse y ser abordada de manera eficaz fuera de la relación analítica, como si no, lo que se conoce como extratransferencia, ha generado periódicamente considerable debate. Seguido de la suposición habitual que considera que si el psicoanalista no "lleva" la transferencia a la relación analítica, el psicoanalista está "contratransferencialmente colaborando con dicha transferencia". Vamos a trazar cuales son los orígenes teóricos de este supuesto técnico y después valoraremos su validez teniendo en cuenta el actual modelo organizador de la transferencia. La proposición que sostengo es que la constante aplicación de este principio durante el encuentro analítico puede ofender la propia experiencia del paciente, dando pie a prestar una excesiva atención hacia el terapeuta, algo de lo que suelen quejarse frecuentemente los pacientes (Fosshage, 1994) y puede ser enormemente perturbador y ofensivo para el paciente y el propio proceso analítico. ${ }^{6}$

\section{Revisión Histórica}

En un primer momento en Estudios sobre la Histeria, Freud (1895) basándose en Breuer y Anna O. plantea que el paciente hace una "falsa conexión" al transferir "a la figura del médico las ideas angustiosas que aparecen debido al contenido del análisis" (p.302). Posteriormente, Freud (1905) re-conceptualiza esta "falsa conexión" como "transferencia" y distingue dos tipos: 1) las que no difieren del modelo más que en la substitución de la figura7 y 2) aquellas que "se benefician de alguna peculiaridad real del propio médico o de las circunstancias", no teniendo como resultado "nuevas impresiones, pero sí ediciones revisadas" (p.116). En el primer caso, la transferencia del paciente distorsiona la realidad del analista ${ }^{8} y$, en el segundo caso, la transferencia del paciente hace uso de las idiosincrasias del analista para apoyar su propio punto de vista (Smith, 2003) - lo que coloquialmente pasó a conocerse como "el gancho del que cuelga el sombrero del paciente"9. Esta segunda distinción es bastante 
sorprendente en tanto que implica la contribución del analista que, aunque con ciertas limitaciones, pasa a ser precursora del futuro reconocimiento que ofrecerá la teoría relacional en el cual el analista es visto como un colaborador activo y cambiante respecto a las transferencias del paciente ${ }^{10}$.

Sin embargo, el modelo clásico o del desplazamiento fue formulado en el momento de pleno apogeo de la ciencia positivista y objetivista y consideraba la transferencia principalmente como percepciones distorsionadas acerca del analista que eran generadas intrapsíquicamente. Clínicamente, el analista neutro, su abstinencia y ser una pantalla en blanco cumplían con el propósito de devolver al paciente sus proyecciones y desplazamientos distorsionados. Según este modelo ${ }^{11}$, cualquier aparición de la subjetividad del analista era vista como un problema contra-transferencial que enturbiaba la lente refleja.

Inicialmente la transferencia era entendida como una de las principales resistencias al tratamiento, impidiendo el recuerdo del material reprimido, más tarde Freud pasa a considerarla como una "poderosa aliada" (1905, p. 117) y pensaba que "cada conflicto debe ser combatido dentro del campo de la transferencia" (1912, p.104, cursivas nuestras) ya que "cuando todo ha sido dicho y hecho, es imposible destruir a alguien en rebeldía o en efigie" (p.108, cursivas en el original). Debido a la conceptualización de que la transferencia se refiere específicamente al "médico" o analista, el análisis de la transferencia se refiere exclusivamente a su aparición dentro de la relación analítica y pasa a ser el foco central del tratamiento psicoanalítico ${ }^{12}$. En otras palabras, por definición la transferencia hace referencia a la relación analítica y su carácter central se enriquece por el paciente y por el trabajo analítico con el concepto de Freud de neurosis de transferencia (Freud, 1914, p. 154). Por lo tanto, el principal campo de batalla del conflicto se hacía explícito en la transferencia hacia el analista. Cuando se detectaba transferencia fuera de la relación analítica, se empleaba un concepto diferente, extra-transferencia, descalificando en parte su importancia. La extratransferencia se entendía como una defensa del propio paciente a que ésta apareciera dentro de la relación analítica, defensa que necesitaba ser interpretada para así permitir que la transferencia fuera llevada a la relación analítica. No hacer esto se pensaba como la colaboración de la contratransferencia del analista con las resistencias a la transferencia del paciente.

Posteriormente, aparecieron las correcciones y los cambios radicales del modelo clásico o del desplazamiento, descritas en detalle por Hoffman (1983). En términos generales, estas correcciones fueron el resultado de un aumento del reconocimiento de la complejidad de la relación analítica y una mayor integración de transformaciones en los paradigmas, desde el objetivismo al constructivismo y desde lo intrapsíquico a la teoría relacional. Respecto al reconocimiento de su complejidad, se descubrió que algunos aspectos de la relación analítica 
no encajaban con la idea de la transferencia como proyecciones y desplazamientos distorsionadores. Por ejemplo, Freud (1912) se refirió a la disposición del paciente para trabajar con el analista como "transferencia positiva inobjetable". A diferencia de esto, Sterba (1934), Zetzel (1956), Greenson (1965) y Loewenstein (1969) hablan de la disposición del paciente a trabajar con el analista en base a la relación real que se da entre ambos, y no cayendo propiamente bajo el marco de la transferencia. Greenson (1971) refirió otros aspectos de la relación terapéutica como elementos de una "relación real", sin implicar distorsiones y, por lo tanto, tampoco transferencia. El concepto de Kohut (1977) de transferencias del objeto del self amplía significativamente el concepto de transferencia centrando la atención no en la repetición del pasado, sello distintivo de la transferencia, sino en el desarrollo actual y posible del self y su cuidado ${ }^{13}$.

En la formulación de su modelo inclusivo, Gill $(1982,1984)$ aportó una definición mucho más amplia de la transferencia refiriéndose ésta a la experiencia que tiene el paciente del propio analista y de la relación analítica. Con esta descripción Gill (1984) supone que la transferencia estaba siempre operativa ${ }^{14}$ en la relación terapéutica, que los comentarios del paciente trataban siempre acerca de la transferencia, y que clínicamente el analista "siempre" debía centrarse en la transferencia. Siguiendo con esto, Gill (1984) destaca la importancia fundamental del análisis de la transferencia, por definición "en la relación analítica", al delinear que cuatro de los cinco aspectos intrínsecos definitorios del psicoanálisis implican transferencia, que son concretamente "lo fundamental del análisis de la transferencia, un analista neutro, la inducción de una neurosis transferencial regresiva y la resolución de dicha neurosis mediante técnicas únicamente interpretativas, o al menos principalmente con interpretaciones" (p.161). Al ser criticado su argumento de que la transferencia en la relación analítica debería ser siempre el eje central del análisis, Gill (1984) "se retractó de haber dicho 'siempre'" en cambio reafirmó "que lo que... debía recibir atención en un primer momento es el significado que las asociaciones tienen para la transferencia" (p. 492), siendo esto transferencia, por definición, en tanto que emerge dentro de la relación analítica ${ }^{15}$.

Desde mi punto de vista, suponer y escuchar gran parte, sino todo, del contenido de los comentarios de un paciente en relación al analista puede perturbar con facilidad la atención hacia el paciente, reducir la importancia de otras relaciones, dar lugar a malentendidos, mientras que al mismo tiempo se origina una asfixiante atmósfera centrada en el analista algo que el paciente puede experimentar en su mejor caso como una evidente singularidad o, en el peor de los casos, una bizarra falta de vinculación (Fosshage, 1994; Wachtel, 2008). Langs (1978) y Gill y Hoffman (1982) han ofrecido un gran número de ejemplos de escucha sistemática del contenido de lo expresado por el paciente, independientemente de su "contenido manifiesto", referente a la relación analítica. Mientras que los ejemplos de Gill y 
Hoffman eran reinterpretaciones de observaciones del proceso, excluyendo evaluaciones de su impacto, los ejemplos que presenta Langs incluyen muy a menudo intercambios que, según mi opinión, demuestran con claridad los problemas antes mencionados.

Mientras que el modelo inclusivo de la transferencia, refiriéndose en su totalidad a la experiencia del paciente respecto a la relación analítica, no es, desde mi punto de vista, suficientemente específico en cuanto a su conceptualización, es evidente que sea lo que sea que esté ocurriendo dentro de la relación analítica tiene importancia incluso cuando no es explícitamente el centro de atención. No obstante, para entender lo que está teniendo lugar dentro de la relación analítica (modelo inclusivo) yo señalé (1994) que la diferenciación entre el contenido y el proceso de comunicación es de una importancia fundamental para poder determinar aquello que conlleva el significado de la transferencia y evitar perturbaciones iatrogénicas por la aplicación sistemática del contenido de los comentarios del paciente a la relación analítica ${ }^{16}$.

Mientras que más adelante Gill abrió la puerta a las contribuciones del analista al terreno analítico, y no únicamente a las del paciente, yo sugiero que la amplia conceptualización de la transferencia que propone Gill no hace hincapié en los patrones "estereotipados" de interacción que Gill (1982) afirma haber encontrado en Freud, como por ejemplo la incorporación que hace Freud de la "transferencia positiva inobjetable" (p.9). Estos patrones de interacción, desde mi punto de vista, pasan completamente a un primer plano con el modelo de transferencia de la siguiente generación, el modelo organizador de la transferencia.

El contexto teórico y clínico en el que fue conceptualizado el modelo del displacer impidió la aparición de la pregunta de si la transferencia podría aplicarse significativamente a relaciones fuera de la relación analítica (la conocida extra-transferencia) ${ }^{17}$ o no. Posteriormente, la pregunta acaba surgiendo plenamente, dando lugar a una considerable controversia acerca de su validez y eficacia (ver Bornstein, Katz \& London, 1984). La controversia, tal y como he intentado aclarar, se originó tras la conceptualización y definición de que la transferencia estaba siempre involucrada en la relación analítica. Por ejemplo, cuando un paciente hace hincapié en un aspecto problemático respecto a una relación fuera de la relación analítica, la posición clínica predefinida ${ }^{18}$ asume que el paciente "en realidad" está hablando de la relación analítica (Gill mantuvo esta posición). Repetiré que el analista entonces es visto como "fracasando" contratransferencialmente si no "lleva" la atención de vuelta a la transferencia dentro de la relación analítica. Desde mi punto de vista, una solución coherente a esta controversia aparece disponible en el desarrollo del posterior modelo organizador de la transferencia. 
Gradualmente y a través de la integración de la ciencia cognitiva, especialmente del trabajo de Piaget (1954) sobre los esquemas, los teóricos psicoanalíticos (Wachtel, 1980; Hoffman, 1983, 1991; Stolorow y Lachmann, 1984/85; Stern, D.N., 1985; Fosshage, 1994; Lichtenberg, Lachmann y Fosshage, 1996; y Stern, D.B., 1997) empleando términos diferentes pero correlativos, conceptualizaron el tercer y más reciente modelo, la transferencia como modelo organizador. Un principio básico de la ciencia cognitiva postula que los seres humanos (junto con otros animales) organizamos cognitiva y afectivamente nuestras experiencias cumpliendo esto una función adaptativa esencial. Basándonos en experiencias vividas, establecemos patrones implícitos y explícitos de organización (también mencionados como principios organizadores, esquemas y construcciones) para gestionar nuestra vida de manera eficiente y eficaz. Una vez que han sido establecidos, estos patrones organizadores consecuentemente influyen nuestra percepción, nuestra organización cognitiva/afectiva y nuestras interacciones interpersonales. Los patrones organizadores funcionan de la siguiente manera: 1) establecimiento de expectativas de acuerdo a experiencias vividas; 2 ) atención selectiva hacia las señales que cumplen nuestras expectativas ${ }^{19}$; 3 ) atribución de significado a estas señales que cumplen nuestras expectativas; y 4 ) interacción interpersonal de forma que se tienda a confirmar y validar dichas expectativas (Fosshage, 1994) ${ }^{20}$. Los patrones de organización son categorías de nuestra memoria a largo plazo.

Una vez establecidas, los patrones de organización varían mucho según su disposición al cambio. Los patrones menos rígidos son influidos por nueva información contrastante y gradualmente adaptados (Piaget, 1954) ${ }^{21}$. Una persona con un patrón más firmemente establecido ni selecciona ni atiende a nueva información contrastante ni tampoco asimila (Piaget, 1954) la información sin cambios en el patrón organizador ${ }^{22}$. Un patrón organizador paranoico, por ejemplo, puede ser rígido e inflexible. Con patrones más rígidos el cambio se pueda dar únicamente a través de la distensión de su activación y la formación de nuevas organizaciones contrastantes (por ejemplo, diferentes imágenes del self con los otros). Dentro de este modelo, los desplazamientos, las proyecciones y otros manejos defensivos se entienden todos ellos como actividades organizadoras (Stolorow, Brandcharft y Atwood, 1987). Por último, nuevas experiencias relacionales pueden registrarse momentáneamente en la memoria a corto plazo 23 , con una propensión a desaparecer si no existe una categoría dentro de la memoria a largo plazo en la cual queden registradas, todo esto supone que el cambio sea más difícil.

Aunque nosotros hablamos de transferencia cuando un patrón organizador es activado dentro de la relación analítica, el modelo organizador ha expandido los ámbitos relacionales en los que se activan los patrones a incluir potencialmente cualquier relación que tenga el paciente ${ }^{24}$. El hecho de que los patrones organizadores se activan y desactivan de forma 
variable en nuestras relaciones, supone a la larga un nuevo supuesto clínico, el análisis de los patrones organizadores donde quiera que hayan sido activados, ya sea en la relación analítica o en otra relación. Este modelo de actividad organizadora por medio de patrones cognitivo/afectivos, en cambio, no da prioridad al énfasis en la relación analítica, sino que expande el alcance analítico para incluir el amplio conjunto de experiencias relacionales del paciente. Mientras que al poner el foco de atención en un patrón activado dentro de la propia relación analítica nos puede dar la oportunidad de un seguimiento detallado de una experiencia inmediata, situar el foco del análisis en un patrón activado en una relación "externa" puede preservar el proceso reflexivo que tiene lugar dentro de la relación analítica ${ }^{25}$. Tanto el seguimiento de cerca como el procesamiento reflexivo facilitan los albores de una toma de conciencia de lo que se experimenta como una "realidad" absorbente que será construida de un modo oscilante a través del uso del patrón organizador de cada uno.

Si el analizando, por ejemplo, está preocupado por una situación con un amigo, el analizando lo que quiere es comprender esta situación y no centrar su atención en el analista. Mientras que esto nos puede resultar obvio e incluso un aspecto de sentido común, el posicionamiento clínico por defecto (Wachtel, 2008) de orientar la atención hacia la transferencia dentro de la relación analítica muy a menudo se encuentra al acecho. En ocasiones los candidatos a analistas admiten con cierta vergüenza y culpa, "No estoy seguro de que esto sea psicoanálisis, no abordé la transferencia. Quizás el paciente sí que hablaba inconscientemente de mí. O, el paciente dice sentirse enfadado con un amigo y el analista comienza a pensar que el paciente está "en realidad" enfadado con el analista y defensivamente habla con lejanía de su verdadero conflicto. Los persistentes supuestos clínicos que provienen por defecto del modelo de la transferencia por desplazamiento, tienden a sobre-enfatizar las operaciones defensivas y dejan pasar inadvertidamente las expresiones del paciente que expresan las preocupaciones inmediatas del paciente $y$, por lo tanto, interfieren en la escucha sintonizada y la comprensión que son los aspectos del buen trabajo clínico.

Cuando un tema organizador, en un momento dado, es activado en una relación fuera del contexto analítico, puede estar o no estar activo dentro de la relación analítica. Normalmente, dentro de nuestra complejidad tenemos un número de experiencias implícitas y explícitas y procesos organizadores ocurriendo simultáneamente. Por ejemplo, cuando el paciente está relatando una experiencia de abuso $^{26}$ cargada de vergüenza, él o ella probablemente está experimentando en ese momento al analista no como un abusador sino como alguien en quien confiar con el que él o ella puede mostrarse vulnerable y abrirse (Fosshage, 1994). Asumir en esta situación que el contenido de las expresiones del paciente 
se dirige a la relación analítica puede afectar con facilidad tanto a la atención del paciente a sus propias expresiones como a la experiencia del proceso en marcha de una relación analítica de confianza. Incluso centrar nuestra atención en esta experiencia de un proceso en curso dentro de una relación analítica segura y de confianza puede alterar nuevas experiencias relacionales. Puede ser un momento de "vivir" dentro de la interacción, de escuchar música, y no tanto de centrar explícitamente nuestra atención, lo que suponga una mayor cura. El motivo de este ejemplo es que el modelo organizador de la transferencia combinado con una distinción entre contenido y proceso provee al analista con un significativo nuevo conjunto de procedimientos clínicos basados teóricamente.

Desde el punto de vista del modelo organizador de la transferencia nosotros, por lo tanto, dentro de un proceso psicoanalítico intentamos iluminar y mejorar la consciencia reflexiva de patrones organizadores desvitalizantes y esencialmente limitantes y sus orígenes relacionales acerca de donde fueron y con quien se activaron. Mientras que la conceptualización de la transferencia se ha referido tradicionalmente a la relación analítica, ahora el modelo organizador se centra en los procesos psicológicos que tienen lugar dentro y fuera de la relación analítica. Para ofrecer un sentido de continuidad seguimos llamando a esta última conceptualización el modelo organizador de la transferencia y para evitar confusiones empleamos muy a menudo el término de patrones organizadores. Cuando hacemos referencia al modelo organizador y empleamos el término transferencia(s), asumimos siempre que las transferencias son potencialmente activadas en cualquier contexto relacional. Por lo tanto, las transferencias hacen referencia a los patrones principales de organización con los que el paciente asimila y construye su experiencia relacional ${ }^{27}$.

\section{Términos Psicoanalíticos Correspondientes}

Con diferentes connotaciones y matices, un gran número de teóricos han dado forma a términos psicoanalíticos que hacen referencia especialmente a patrones de procesamiento ya constituidos. Algunos de los más conocidos son el mundo representacional (Sandler y Rosenblatt, 1962); modelos internos de trabajo (Bowlby, 1973); esquemas (Wachtel, 1980); principios o patrones de organización (Stolorow y Lachmann, 1984/1985; Fosshage, 1994); representaciones de interacciones generalizadas o RIGs (Stern, 1985); creencias patológicas (Weiss y Sampson, 1986); representaciones mentales (Fonagy, 1993); estructuras de interacción (Beebe y Lachmann, 2002); expectativas (Lichtenberg, Lachmann y Fosshage, 1996); temas de organización (Sander, 1977). Por razones de consistencia tenemos una tendencia a emplear el término de patrones de organización o patrones organizadores. 


\section{El Uso de la Subjetividad del Analista}

El avance en la integración del cambio en los dos paradigmas, del objetivismo al constructivismo y de la teoría intrapsíquica a la teoría relacional, abrió la puerta al actual y extenso uso de la subjetividad del analista para el entendimiento del ámbito (sistema) de la diada analítica, la consiguiente evolución del término conocido previamente como matriz transferencia-contratransferencia. Mientras que Freud definió la contratransferencia como las reacciones patológicas a la transferencia que debían ser identificadas y dominadas para "limpiar" al analista como lente refleja, los autores relacionales de todas las corrientes (Fosshage, 2003) han expandido el uso de un amplio abanico de experiencias subjetivas del analista como potencialmente esclarecedoras del paciente y del proceso interactivo. Los enactments (Bromberg, 1998; Davies, 2004; Fosshage, 1995a; Stern, 1997), procesos procedimentales implícitos (Stern, y cols. 1998; Fosshage, 2011b), diferentes perspectivas de escucha (Fosshage, 1995b, 2011a) y nuevas experiencias relacionales (Aron y Atlas, 2015; Bass, 2012; Davies, 1994; Shane, Shane y Gales, 1997; Summers, 2012) subrayan todos ellos la participación y el uso de la subjetividad del analista en la facilitación de la acción terapéutica.

Mientras este amplio uso de la experiencia del analista aporta inestimablemente un mayor acceso a los mundos de experiencia de nuestros pacientes y al campo de interacción analítica, hacemos también un llamamiento a la prudencia, por su potencial de poder reforzar, una vez más, la posición clínica por defecto de poner atención exclusivamente en la transferencia dentro de la relación analítica. Por ejemplo, en la última reunión de IARPP en Toronto advertimos que todos los ponentes se centraron exclusivamente en la "transferencia" dentro de la relación analítica. No se hizo mención ninguna a transferencias fuera de la relación analítica. Nosotros defendemos que esta atención exclusiva está basada en la errónea suposición de que todos los principales patrones organizadores del paciente necesariamente se activarán en la relación analítica. Esta suposición imposibilita la contribución del analista a la experiencia del paciente y cómo la transferencia es co-creada y co-construida de manera variable dentro del campo intersubjetivo. Creemos, por lo tanto, que una atención exclusiva en la transferencia dentro de la relación analítica es posiblemente muy limitante para un análisis riguroso. En cambio, lo que nosotros proponemos, basado en el modelo organizador de la transferencia, es que cuando aparecen patrones organizadores asociados a un trauma, muy restrictivos o desvitalizadores - ya sea dentro o fuera de la relación analítica - debemos analizar dónde y cuándo se han desencadenado estos patrones. Durante estas situaciones en las cuales la activación de dichos patrones ocurre fuera de la relación analítica, el esfuerzo reflexivo conjunto dentro de la relación analítica (el proceso), supondrá muy probablemente una nueva e importante experiencia relacional. 


\section{Procesamiento Implícito y Explícito en la Formación de Patrones Organizadores}

Importantes investigaciones dentro de la ciencia cognitiva han documentado empíricamente que existen dos tipos de procesos cognitivos diferentes, pero complementarios, que están involucrados en la adquisición de conocimiento cognitivo y relacional y destrezas (ver Sun, 2002, para una revisión sobre este tema). Estos procesos afectivo/cognitivos aparecen en dos niveles de consciencia mientras estamos despiertos - el implícito o no-consciente y el explícito o consciente (ver Fosshage, 2005, 2011b para revisión). El implícito hace referencia al procesamiento no-declarativo y el explícito al procesamiento declarativo. La investigación del sueño ha documentado que el soñar, durante las fases REM y no-REM, se mantiene el procesamiento afectivo/cognitivo durante los ciclos del sueño y se contribuye así a la consolidación de patrones organizadores, Drew Weston (2006) escribe, "Los investigadores actuales han identificado que la mayor parte del procesamiento ocurre a un nivel no consciente, ya que el cerebro procesa información en paralelo" (p. 244).

Muchos científicos cognitivos postulan acerca de dos formatos de codificación y procesamiento (Epstein, 1994). Por ejemplo, Paivio $(1971,1986,2007)$ propone la existencia de codificación simbólica imaginística y verbal. Bucci añade un tercer formato, el procesamiento subsimbólico. Incorporándose esto a inconsciente dinámico de Freud, estos hallazgos aumentan de manera exponencial el volumen del procesamiento inconsciente.

A diferencia del inconsciente dinámico de Freud que supone conflicto y defensas y al actual énfasis en la disociación, que supone mantener y proteger estados separados del Self, el procesamiento implícito y la memoria implica procesamiento inconsciente (o no-consciente) que incluye una gran cantidad de aprendizajes. La memoria implícita involucra varios sistemas de memoria - aprendizajes de habilidades, priming ${ }^{28}$, condicionamiento clásico, modelado y aprendizaje procedimental. Recientemente el aprendizaje procedimental implícito se ha ampliado al aprendizaje de patrones relacionales (Clyman, 1991; Davis, 2001; Grigsby \& Hartlaub, 1994), lo que el BCPSG ha pasado a denominar "conocimiento relacional implícito" (Grupo de Boston para el Estudio de los Procesos de Cambio, 2002, 2005, 2008; D. N. Stern, y cols., 1998). Mientras que ciertos procedimientos de interacción relacional se transmiten explícitamente $y$, por consiguiente, se vuelven automáticamente procedimientos relacionales implícitos (por ejemplo, conductas de buena educación), otros procedimientos de interacción (patrones organizadores) son aprendidos a un nivel inconsciente (lo que Stolorow y Atwood, 1991, denominan como el inconsciente pre-reflexivo) y pueden quedar "automatizados" o pasar por primera vez a un nivel consciente, por ejemplo, dentro de análisis. 
Las investigaciones ofrecen una amplia evidencia de diferencias neuroanatómicas entre los dos sistemas, el procesamiento implícito/no-declarativo involucra al hemisferio derecho y, por otro lado, el procesamiento explícito/declarativo está vinculado al hemisferio izquierdo (Schore, 2003, pp. 52-53). El concepto de "modelos mentales implícitos" surge de la investigación experimental dentro de la psicología cognitiva, converge con el concepto de la neurociencia de mapas o redes neurales de memoria (Nelson, 1986; Edelman, 1987; Leven, 1991) así como también lo hace con el término psicoanalítico de patrones organizadores, al dar forma al nivel implícito.

Hagamos una breve referencia ahora a la acción terapéutica, actualmente el psicoanalista aborda conflictos pesados y fuerzas represoras (el inconsciente dinámico de Freud), apariciones de estados disociados del Self, actitudes explícitas restrictivas y procedimientos relacionales implícitos desvitalizadores. Las actitudes explícitas (lo que son los patrones organizadores explícitos) son modificables directamente al dirigir nuestra atención explícita/declarativa. Los modelos mentales implícitos (patrones organizadores implícitos) pueden modificarse de manera variable a través de dos procesos: (1) por acomodación gradual o modificación de las expectativas por medio de nuevas experiencias (no requiere de atención explícita) y (2) un aumento de la capacidad para desactivar, al adoptar una actitud consciente reflexiva, la correspondiente frecuencia de activación disminuida y el establecimiento de modelos implícitos opuestos, a través de nuevas experiencias procedimentales implícitas (Fosshage, 2005, 2011b).

Resumiendo mi comentario de esta noche, he tratado de presentar la evolución de 3 modelos fundamentales de entender la transferencia, el del displacer, el inclusivo y el modelo organizador.

Estos modelos tienen implicaciones clínicas de gran alcance. Durante la presentación de esta noche me he centrado más en el principio técnico del psicoanálisis tradicional que explica que el foco principal, sino exclusivo, se centra en la transferencia de la relación analítica. Esto sigue el supuesto común de que si el psicoanalista no "lleva" la transferencia a la relación analítica, estará entonces "contra-transferencialmente colaborando con la transferencia". El origen teórico de este supuesto clínico está ligado a la primera conceptualización de Freud y a la definición de trasferencia que conlleva desplazamientos hacia el analista. El posterior desarrollo de Freud del concepto de neurosis de transferencia reforzó el foco principal en la relación analítica. Mi hipótesis es que el constante uso de este supuesto durante el encuentro psicoanalítico puede derivar fácilmente en la desestimación de las experiencias expresadas por el paciente, orientando la atención demasiado hacia el analista, algo de lo que muy a menudo se quejan los pacientes, alterando y perjudicando al paciente y al propio proceso analítico. 
La integración de la ciencia cognitiva ha guiado la actual conceptualización del modelo organizador de la transferencia que nos ha provisto de un entendimiento crucial y ampliado de la transferencia, que se presenta en la organización de la experiencia. Dentro de este modelo, las transferencias se refieren a los patrones fundamentales de organización con los cuales el paciente asimila y construye su experiencia relacional. El hecho de que los patrones de organización se activan y desactivan de forma variable dentro de las relaciones en general respalda un nuevo supuesto clínico, este es, el de analizar los patrones organizadores allá donde se activen, ya sea dentro de la relación analítica o en cualquier otra relación. Por ello, este modelo de actividad organizadora basada en patrones cognitivo/afectivos no ofrece prioridad al énfasis en la relación analítica, sino que expande el alcance analítico incluyendo un registro amplio de las experiencias emocionales del paciente.

\section{REFERENCIAS}

Aron, L. and Atlas, G. (2015). Generative enactment: Memories from the future. Psychoanalytic Dialogues, 25:309-324.

Atwood, G. and Stolorow, R. (1984). Structures in Subjectivity. Hillsdale, NJ: The Analytic Press.

Bass, A. (2012). Negotiating otherness: The analyst's contribution to creating new ways of being and relating in the analytic process: Commentary on paper by Frank Summers, Psychoanalytic Dialogues., 11: 162-170.

Beebe, B. and Lachmann, F. (2002). Infant Research and Adult Treatment. Hillsdale, NJ: The Analytic Press.

Bornstein, M., Katz, J. and London, N. (1984). Editors, Commentaries on Merton Gill's Analysis of Transference, Psychoanalyitc Inquiry, Vol. 4, No. 313-523.

Boston Change Process Study Group (BCPSG): Bruschweiler-Stern, N., Harrison, A., Nahum, J., Sander, L., Stern, D. \& Tronick, E. (2002). Explicating the implicit: The local level and the microprocess of change in the analytic situation. Internat. J. Psycho-Anal., 83:1051-1062.

Boston Change Process Study Group (2005). The "Something More" than interpretation. Journal of the American Psychoanalytic Association, 53:3, 693-729.

Boston Change Process Study Group (2008). Forms of relational meaning: Issues in the relations between the implicit and reflective-verbal domains. Psychoanalytic Dialogues, Vol. 18, 2, 125-148.

Bowlby, J. (1973). Attachment and Loss. Vol. 2: Separation. New York: Basic Books.

Bromberg, P.M. (1998). Standing in the spaces: Essays on clinical process, trauma, and Dissociation. Hillsdale, NJ: Analytic Press.

Bucci, W. (1997). Psychoanalysis and Cognitive Science: A Multiple Code Theory. New York: Guilford Press. 
Clyman, R. B. (1991). The procedural organization of emotions: A contribution of cognitive science to the psychoanalytic theory of therapeutic action. J. Amer. Psychoanal. Assn., 39(Suppl.):349-382.

Davis, J. T. (2001). Revising psychanalytic interpretations of the past: An examination of declarative and non-declarative memory processes. Internat. J. Psycho-Anal., 82:449-462.

Davies, J. (1994). Love in the afternoon. Psychoanalytic Dialogues, 4, 2: 153-170.

Davies, J. (2004), Whose bad objects are these anyway? Repetition and our elusive love affair with evil. Psychoanalytic Dialogues, 14:711-732.

Edelman, G. (1987), Neural Darwinism: The Theory of Neural Group Selection. New York: Harper \& Row.

Epstein, S. (1994), Integration of the cognitive and the psychodynamic unconscious. Amer. Psychol., 8:709-724.

Fosshage, J. (1994). Toward reconceptualizing transference: theoretical and clinical considerations. Int J. of Psycho-Anal., 75, 2: 265-280.

Fosshage, J. (1995a). Interaction in Psychoanalysis: A Broadening Horizon.Psychoanalytic Dialogues, 5(3):459-478.

Fosshage, J. (1995b). Countertransference as the analyst's experience of the analysand: Influence of listening perspectives. Psychoanalytic Psychology, 12(3), 375-391.

Fosshage, J. (1997). The organizing functions of dream mentation. Contemporary Psychoanalysis, Vol. 33, No. 3, 429-458.

Fosshage, J. (2003). Contextualizing self-psychology and relational psychoanalysis: Bidirectional influence and proposed syntheses. Contemporary Psychoanalysis, Vol. 39, No. 3, 411-448.

Fosshage, J. (2005). The explicit and implicit domains in psychoanalytic change. Psychoanalytic Inquiry, Vol 25, 4: 516-539.

Fosshage, J. (2011a). The use and impact of the analyst's subjectivity with empathic and other listening/experiencing perspectives. Psychoanalytic Quarterly, Vol. LXXX, 1, 139-160.

Fosshage, J. (2011b). How do we "know" what we "know?" And change what we "know?" Psychoanalytic Dialogues, 21, 1: 55-74.

Fosshage, J. (2013). Forming and transforming self-experience. International Journal of Psychoanalytic Self Psychology, Vol. 8, No. 4, 437-451.

Freud. S. (1895). Studies on Hysteria. S. E. 2.

Freud, S. (1905). Fragment of an analysis of a case of hysteria. S.E. 7: 7- 122.

Freud, S. (1912). The dynamics of transference. S.E. 12: 97-108.

Freud, S. (1914). Remembering, repeating and working-through. S.E. 12:145-156.

Gill, M. (1982). Analysis of Transference I: Theory and Technique. New York: International Universities Press. 
Gill, M. (1984). Transference: a change in conception or only in emphasis? Psychoanalytic Inquiry , 4, 3: 489-523.

Gill, M. and Hoffman, I.Z. (1982). Analysis of Transference II. New York: International Universities Press.

Greenberg, J. and Mitchell, S.A. (1983). Object Relations in PsychoanalyticTheory. Cambridge: Harvard University Press.

Greenson, R. (1965). The working alliance and the transference neurosis. Psvchoanalytic Quarterly, 34:155-181.

Greenson, R. (1971). The real relationship between the patient and the psychoanalyst. In The Unconscious Today, ed. M. Kanzer. New York: International Universities Press.

Grigsby, J. \& Hartlaub, G. (1994). Procedural learning and the development and stability of character. Percept. Motor Skills, 79:355-370.

Hobson, J. A. (2013). Ergo sum ergo: Toward a psychodynamic neurology. Contemporary Psychoanalysis, 49:2. 142-164.

Hoffman, I. (1983). The patient as interpreter of the analyst's experience. Contemporary Psychoanalysis, 19: 389-422.

Hoffman, I. (1991). Discussion: toward a social-constructivist view of the psychoanalytic situation. Psychoanalytic Dialogues, 1: 74-105.

Kohut, H. (1977). The Restoration of the Self. New York: International Universities Press.

Langs, R. (1978). Technique in Transition. New York: Jason Aroson, Inc.

Leven, F. (1991). Mapping the Mind. Hillsdale, NJ: The Analytic Press.

Lichtenberg, J., Lachmann, F. and Fosshage, J. (1996). The Clinical Exchange: Technique from the Standpoint of Self and Motivational Systems. Hillsdale, NJ: The Analytic Press.

Lowenstein, R.M. (1969). Developments in the theory of transference in the last fifty years. International Journal of Psycho-Analysis, 50:583-588.

Mitchell, S. (1988). Relational Concepts in Psychoanalysis. Cambridge, MA: Harvard University Press.

Nelson, K. (1986). Event Knowledge. Hillsdale, NJ: Lawrence Erlbaum Associates.

Paivio, A. (1971). Imagery and Verbal Processes. New York, Holt, Rinehart \& Winston. Reprinted 1979, Hillsdale, NJ: Lawrence Erlbaum Associates.

Paivio, A. (1986). Mental Representations: A Dual Coding Approach. New York: Oxford Universities Press.

Paivio, A. (2007). Mind and Its Evolution: A Dual Coding Theoretical Approach Mahway, NJ: Lawrence Erlbaum Associates.

Piaget, J. (1954). The Construction of Reality in the Child. New York: Basic Books. Orange, D. (1995). Emotional Understanding: Studies in Psychoanalytic Epistemology. New York: Guilford Press. 
Sander, L. (1977). The regulation of exchange in the infant-caretaker system and some aspects of the context-content relationship. In: Interaction, Conversation and the Development of Language, ed. M. Lewis \& L. Rosenblum. New York: Wiley, pp. 133-156.

Sandler, J. \& Rosenblatt, B. (1962), The concept of the representational world. The Psychoanalytic Study of the Child, 17:128-145. New York: International Universities Press.

Schachter, J. (2002). Transference: Shibboleth or Albatross? Hillsdale, NJ:The Analytic Press.

Schore, A. (2003), Affect Regulation and the Repair of the Self. New York: Norton.

Shane, M., Shane, E., and Gales, M. (1998). Intimate Attachments: Toward a New Self Psychology. New York: Guilford Press.

Smith, H.F. (2003). Analysis of transference: A North American perspective. Int. J. Psycho-Anal., 84: 1017-1041.

Sterba, R. (1934). The fate of the ego in analytic therapy. International Journal of PsychoAnalysis, 15: 117-126.

Stern, D. N. (1985), The Interpersonal World of the Infant. New York: Basic Books.

Stern, D.N, Sander, L., Nahum, J., Harrison, A., Lyons-Ruth, K., Morgan, A., Bruchweiler-Stern, N. \& Tronick, E. (1998). Non-interpretive mechanisms in psychoanalytic therapy: The "something more" than interpretation. Internat. J. Psycho-Anal., 79:903-921.

Stern, D.B. (1997). Unformulated Experience: From Dissociation To Imagination In Psychoanalysis. Hillsdale, NJ: The Analytic Press.

Stolorow, R. \& Atwood, G. (1992), Contexts of Being: The Intersubjective Foundations of Psychological Life. Hillsdale, NJ: The Analytic Press.

Stolorow, R. \& Lachmann, F. (1984/85). Transference: the future of an illusion. The Annual of Psychoanalysis, 12/13:19-37.

Stolorow, R., Brandchaft, B., and Atwood, G., (1987). Psychoanalytic Treatment, An Intersubjective Approach, Hillsdale, N.J.: The Analytic Press.

Summers, F. (2012). Creating new ways of being and relating. Psychoanalytic Dialogues 22: 143161.

Sun, R. (2002). Duality of the mind. Lawrence Erlbaum Associates, Mahwah, NJ.

Wachtel, P.F. (1980). Transference, schema and assimilation: The relevance of Piaget to the psychoanalytic theory of transference. The Annual of Psychoanalysis, 8: 59-76.

Wachtel, P.L. (2008). Relational Theory and the Practice of Psychotherapy. New York: Guilford Press.

Weiss, J. \& Sampson, H. (1986), The Psychoanalytic Process. New York: Guilford Press.

Westen, D. (2006), Implications of research in cognitive neuroscience for psychodynamic psychotherapy. In Oxford Textbook of Psychotherapy, ed.s G.O. Gabbard, J.S. Beck, \& J. Holmes. 
Westen, D. and Gabbard, G. O. (2002). Developments in cognitive neuroscience: Il. Implications for theories of transference. J. of the Amer. Psychoanalytic Asso., 50/1: 99-134.

Zetzel, E.R. (1956). Current concepts of transference. International Journal of Psycho-Analysis, 37:369-376.

Original recibido con fecha: 20/4/2016 Revisado: 19/06/2016 Aceptado: 30/6/2016

NOTAS Y COMENTARIOS AL TEXTO:

${ }^{1}$ El Prof. James I. Fosshage comenta sobre el título del presente trabajo que éste fue escrito dentro de un grupo de escritura que él dirige en donde varias personas del grupo hablaban sobre la transferencia y de que deberían de hacer un trabajo sobre ese tema. Esta es la primera parte de ese trabajo, escrito por Fosshage, en el cual integra la contribución de otras ciencias ya que la teoría de los sistemas, la teoría cognitiva social, la ciencia cognitiva y la neurociencia, todas contribuyen a lo que él llama el modelo organizador de la transferencia. Él comenta: Primero hablé del modelo organizador de la transferencia en mi trabajo sobre la transferencia publicado en el International Journal of Psychoanalysis, 1994.

${ }^{2}$ Comentario de J. Fosshage: El modelo de desplazamiento es de Freud y el modelo inclusivo (en el original inglés se denomina totalist) de Merton Gill.

3 Comentario de J. Fosshage: Utilizo dos términos: el co-crear y co-construir. El por qué es co-crear interactivamente juntos es porque paciente y analista están co-creando juntos y cada analista y paciente coconstruyen su propio mundo. Hay dos maneras fundamentales de co-construcción: la primera es perceptualmente "cómo me siento y organizo mi percepción" y la segunda es interactivamente "cómo percibo la interacción".

${ }^{4}$ Comentario de J. Fosshage: Esto es muy importante, escucharán que quito el término de la transferencia sutilmente para que no la reconozcan, uso el término de patrones organizadores porque tiene la ventaja de intercambiar términos como transferencia y contratransferencia cuando estamos hablando en el campo relacional.

${ }^{5}$ Comentario de J. Fosshage: Muy importante, hacemos supuestos residuales que se mantienen como patrones automatizados que usamos automáticamente y aparecen de nuevo. Nos proveen con un consistente sentido del self y por lo tanto es difícil cambiarlo, como cuando pegamos a la pelota con una raqueta de tenis una y otra vez de la misma manera. No es tan fácil, es por eso que los cambios en el acercamiento clínico es más complicado que adaptarse a cambios a nivel teórico.

${ }^{6}$ Comentario de J. Fosshage: Si el paciente quiere hablar de otras relaciones del exterior y el analista sigue insistiendo en escucharlo como parte de la relación analítica, esto va a ofender a la propia experiencia del paciente lo cual es perturbante y anti-terapéutico, esa es la tesis.

${ }^{7}$ Comentario de J. Fosshage: La persona substituye al analista por la figura del padre o la madre.

${ }^{8}$ Comentario de J. Fosshage: En el modelo organizador nunca uso la palabra distorsión.

${ }^{9} \mathrm{~N}$. de T: traducción al español de la expresión "the hook the patient's hangs his hat on" que simboliza la dependencia sobre algo o alguien, en este caso del paciente respecto del analista.

${ }^{10}$ Comentario de J. Fosshage: Siempre está en cuestión lo que nosotros contribuimos también, a menos que sigamos pensando en la teoría de campo, no llega a nosotros esa información y el análisis se mantiene en el modelo unipersonal en donde todo es proveído por el paciente. Si esperamos a que todo venga del paciente, el analista se verá siempre de una manera cambiante con respecto a las transferencias del paciente. Desde nuestra perspectiva contemporánea, la llamada transferencia es co-creada y co-construida por ambos. Esto es a lo que yo me refiero como una pregunta fundamental del trabajo analítico, de quién contribuye a qué, tanto a la 
experiencia del paciente como a la del analista. No podemos hacerlo 'objetivamente' porque siempre pasa por nuestra subjetividad, pero lo intentamos lo mejor que podemos.

${ }^{11}$ Comentario de J. Fosshage: Este es un modelo elegante y tiene sentido. El origen de la pantalla en blanco proviene del mundo intrapsíquico del paciente y no queremos nosotros moldearlo o modularlo, por lo que lo que hacemos es reflejárselo de vuelta.

12 Comentario de J. Fosshage: Asegúrense de entender bien esta frase. Es dentro de la definición de transferencia la que se refiere al analista y, por tanto, por eso siempre nos enfocamos en el analista. Dije que en la definición de Freud sobre la transferencia que el desplazamiento va hacia el analista, como ocurre en la relación analítica. Por tanto, ese es el origen de por qué es siempre en analista. Nos referimos al analista cuando hablamos de transferencia y de la experiencia del paciente acerca del analista.

${ }^{13}$ Comentario de J. Fosshage: La complejidad de la relación analítica y nuestro reconocimiento de ella fue incrementando. Entonces, iban surgiendo más aspectos de la relación de las que no podemos ubicarlas como parte de la transferencia. Noten que todo contribuye a la gradual emergencia del campo relacional o de la teoría intersubjetiva. A causa de esta complejidad, ahora la relación analítica es parte de ambos participantes. Se mueve al campo relacional por la complejidad de la relación.

${ }^{14}$ Comentario de J. Fosshage: Es operativa porque, por definición, cuando el paciente está dentro de la sesión analítica ya está experimentando cualquier cosa con el analista y esto ya es transferencia. Les haré una pregunta, si un paciente está sintiendo al analista en un momento particular como frío y poco responsivo y si el $98-99 \%$ de los pacientes sienten al analista de esta manera, ¿es esto transferencia? Acorde a Gill sí lo es, porque sus sentimientos son experimentados en la relación analítica, pero yo sugeriría que no hay que interesarse en eso porque sólo nos muestra que el paciente puede experimentar o sentir las cosas de tal manera que hay una validación consensual sobre ello. Este sería un ejemplo de la habilidad del paciente para proceder igual que muchos otros procederían objetivamente y consensualmente validado. Tendríamos que ver si esto tiene un significado particular para el paciente. Ésta percepción simplemente no es suficientemente específica, desde mi punto de vista, para que sea importante en el análisis. Gill lo describe como una definición totalista de la transferencia.

${ }^{15}$ Comentario de J. Fosshage: Entonces, Gill y Hoffan en 1993 publicaron un libro sobre transferencia donde dieron varios ejemplos clínicos que grabaron y mostraban cómo el terapeuta seguía omitiendo que el paciente estaba hablando sobre el analista. Gill asume que siempre está ocurriendo en la relación analítica, él escucha todo como relacionado hacia la relación analítica. Robert Langs de Nueva York solía hacer lo mismo, y si quieren alocarse deberían de leerlo, porque lo escucha todo como si el paciente siempre estuviera hablando de nosotros (los analistas). Puede crear un estado paranoide en el analista y desde la posición del paciente el analista siempre está hablando de sí mismo. También ocurre algo raro acerca del paciente que llega a consulta porque tiene problemas en sus relaciones externas y acaba repentinamente hablando de los problemas que le plantea el analista y le dirá el paciente: "¿acaso no tengo problemas afuera como para estar hablando de problemas aquí adentro?"

${ }^{16}$ Comentario de J. Fosshage: La diferencia está en ver si es el contenido que trae el paciente es aplicable a la relación analítica o es el proceso de comunicación. Yo siempre planteo una situación en donde el paciente está hablando con el analista acerca de una vergonzosa experiencia de abuso y, en ese momento, muy probablemente el paciente no siente al analista como abusivo, lo cual es atribuible al contenido del paciente. El paciente siente al analista como a un individuo digno de confianza, con lo cual puede comunicar su experiencia. Entonces, el proceso de comunicación es lo que tiene sentido, entre ambos.

${ }_{17}$ Comentario de J. Fosshage: Siempre fue definido dentro de la relación analítica. Surge el problema de si podemos o no enfrentar la transferencia en otras relaciones.

${ }^{18}$ Comentario de J. Fosshage: Se refiere al procedimiento usual del clínico, el procedimiento que solemos pensar automáticamente.

${ }^{19}$ Comentario de J. Fosshage: Otro ejemplo sería la desatención selectiva de no escuchar lo que no va con nuestra experiencia. Un ejemplo claro es el de un paciente con un patrón organizador armado por la idea de " $\mathrm{A}$ nadie le importo" basado en sus experiencias primarias. En la relación analítica o en otra relación, el paciente sentirá que hay diveras expresiones para mostrar el interés o preocupación, como en las relaciones amorosas, y quizá en la relación analítica nosotros hagamos alguna muestra de interés o cariño- verbalmente o no- y nos CelR Vol. 10 (2) - Junio 2016 ISSN 1988-2939-www.ceir.info

(c) Derechos reservados/Copyright de Clínica e investigación Relacional y los autores. Prohibida la reproducción total o parcial sin autorización expresa. Este material es para uso científico y profesional exclusivamente y puede contener información clínica sensible. Los editores no se responsabilizan de los contenidos de los autores. Dirigir las consultas sobre derechos y autorizaciones a ceir@psicoterapiarelacional.es 
haga sentir involucrados al dar esa muestra de interés. ¿Cómo es que un paciente no recuerde esto? Estas expresiones de interés, preocupación o cuidado son selectivamente desatendidas por el paciente a causa de los patrones organizadores que le sesgan. Lo que presenta el paciente es una serie de categorías de la memoria de largo plazo que dicen "No me siento querido"; "No le importo a nadie". Lo que sucede es que estas respuestas de interés o cuidado son desatendidas o las reconoce como una expresión de preocupación y se almacena la experiencia en la memoria de corto plazo y no llega a establecerse en la memoria a largo plazo. Estas experiencias se pierden porque no corresponden a las categorías primarias de los patrones organizadores. Esto es muy importante porque ahora comprendemos mejor lo que está pasando ahí y no es que el paciente sea una persona que quiera rechazar o que no muestre interés por las relaciones, o que no hayan sido capaces de ver esas respuestas, esas experiencias, ya que sucede que estas experiencias se desvanecen por el poco impacto que tuvo sobre la memoria a largo plazo para poder consolidarse. Si todas las personas tenemos una gran colección de experiencias, incluyendo por lo menos unas cuentas experiencias buenas, entonces ¿por qué estas no toman lugar? Hay dos razones para entender porque no toman lugar y es por ello que alguien llega para pedir análisis, ya que de esa manera podemos ayudar al paciente a entender lo que están dejando pasar y porque continúan sin percibirlo, pasándolos por alto.

${ }^{20}$ Comentario de J. Fosshage: Sobre la interacción siempre doy este ejemplo: Digamos que voy a almorzar con un colega que no conozco muy bien y ese día en particular- por lo que sea que me ocurría- de pronto empiezo a tener sentimientos de expectativas como "Sabes, no creo que le agrade tanto". Me siento en el restaurante y él entra por la puerta y, por lo tanto, me muestro un tanto aversivo de manera protectora. Él lee mi aversión y piensa "Oh, a Jim no le agrado tanto", él se vuelve aversivo y es así como esto opera, impactando en nuestra interacción y a la validación de esas expectativas. Otro ejemplo de experiencia sería el que estuviera sentado en el café, esperando a la otra persona, y comienzo a pensar en buenos sentimientos como "Realmente me agrada esta persona"; "Realmente estoy emocionado por verlo"; "Creo que le agrado también". Entonces, cuando aparece por la puerta, tengo esta gran sonrisa y él piensa "Oh, a Jim le agrado" y me da una sonrisa de vuelta y tenemos un buen almuerzo. Parecido a la profecía autocumplida, este es un entendimiento más específico de cómo esto opera de forma cognitiva. Tiene un efecto inmediato porque en ocasiones el paciente y analista no van a congeniar con la respuesta o clave, entonces el analista dice no reconocer esa respuesta o clave y en otras ocasiones puede que ambos estén de acuerdo con las claves o respuestas provenientes del analista pero difiriendo en el significado de las mismas.

${ }^{21}$ Comentario de J. Fosshage: Algunos patrones organizacionales son menos rígidos y se pueden acomodar a nueva información.

${ }^{22}$ Comentario de J. Fosshage: Lo que hacen es que cambian la información para que encajen en el patrón organizador, en vez de que la información cambie el patrón organizador.

${ }^{23}$ Comentario de J. Fosshage: Para los patrones rígidos, el modo que tienen de cambiar es por medio de la consciencia reflexiva y con ella viene la habilidad para interceder y desactivar el patrón organizador. Si pudiera decir: "Aquí viene de nuevo" -ese patrón organizador- "Estoy familiarizado con ello"; "Sé el origen de ello" y me permito dejarlo ir y ver si puedo experimentar el mundo de una forma distinta. Así es como desactivamos los patrones desorganizadores, hemos adquirido una nueva habilidad. De otra forma, la única manera en que uno puede cambiar es por medio de la repetición de nuevas experiencias una y otra vez para que se establezca un tema experiencial en contraste con el patrón organizador. El problema con esto es que esta nueva experiencia es almacenada en la memoria de corto plazo, por lo que termina disipándose. El paciente y el analista pueden verse frustrados al tratar de crear una nueva experiencia que nunca llegan a establecerse. Desde mi punto de vista en base a lo que dice el grupo de Boston es que un cambio ocurre en un nivel de conocimiento implícito y que las nuevas experiencias relacionales formarán nuevo conocimiento relacional, pero yo opino que no hay que ir tan deprisa, porque si un patrón organizador sigue ahí y no es tan sencillo modificarlo, considero que el grupo de Boston subestima la importancia de la exploración porque me parece de suma importancia la concientización de esos rígidos patrones organizativos. Esto es lo que hacemos, por ejemplo, si el paciente tiene una tendencia en caer sobre estados mentales depresivos y de desesperanza se debe a que siempre se ha sentido así desde que fue creciendo. Al identificar este patrón organizador en su estado mental y sus orígenes relacionales, le permite al paciente hacerse consciente de ellos como temas experienciales de su realidad. La pregunta fundamental sería algo así como: ¿Recuerdas algún momento en específico en donde comenzaste a sentirte de CelR Vol. 10 (2) - Junio 2016 ISSN 1988-2939 - www.ceir.info

@ Derechos reservados/Copyright de Clínica e investigación Relacional y los autores. Prohibida la reproducción total o parcial sin autorización expresa. Este material es para uso científico y profesional exclusivamente y puede contener información clínica sensible. Los editores no se responsabilizan de los contenidos de los autores. Dirigir las consultas sobre derechos y autorizaciones a ceir@psicoterapiarelacional.es 
esa manera? Esa simple pregunta de ¿cómo desarrollaste esa actitud hacia ti mismo de que eres perezoso o tonto? Y el paciente responderá: "Oh, ¿entonces esto no es real?" esto incrementar la consciencia reflexiva.

${ }^{24}$ Comentario de J. Fosshage: El término de que tienes "tendencia" a, es muy importante aquí.

${ }^{25}$ Comentario de J. Fosshage: En la relación analítica la ventaja es que podemos rastrearla mientras ocurre. La desventaja cuando es activada en una relación externa, protege el espacio reflexivo entre analista y paciente por lo que juntos se enfocan en esto. Por ejemplo, si es activada en la relación analítica el paciente podría decir: "en realidad a ti no te importo" lo cual refleja un sentimiento de que al analista no le importa y tiende ser debilitada la capacidad para reflexionar sobre ello, queda rasgada por el enojo de la situación. Es rasgada por este patrón organizador. Por eso si ocurre en una relación externa es más fácil reflexionar sobre ello.

${ }^{26}$ Comentario de J. Fosshage: Incluso si en la relación analítica hay una experiencia de seguridad, significa el proceso de comunicación tiene un significado para la relación analítica. Quizá no deseemos lidiar con ello en ese momento y hablarlo después. Creo que todos saben que algunas veces nos sobre-enfocamos con palabras que pueden romper una experiencia sanadora que estaba ocurriendo en la relación analítica. En esas situaciones debemos vivir la experiencia y después hablar de ello. No es distinto de nuestras relaciones sociales, de nuestras relaciones amorosas; necesitamos vivir el romance, vivir las amistades y después podremos hablar de ello.

${ }^{27}$ Comentario de J. Fosshage: Esta es la nueva definición de transferencia acorde al patrón organizador. Actualmente considero que hay que ampliarlo también a la experiencia relacional del paciente acerca de la transferencia.

${ }^{28} \mathrm{~N}$. de T: Priming es el término utilizado dentro de la Neuropsicología y Psicología de la Atención que atiende a la influencia que la experiencia con un estímulo previo puede tener en el procesamiento de un estímulo posterior. El priming está directamente relacionado con la memoria implícita. 